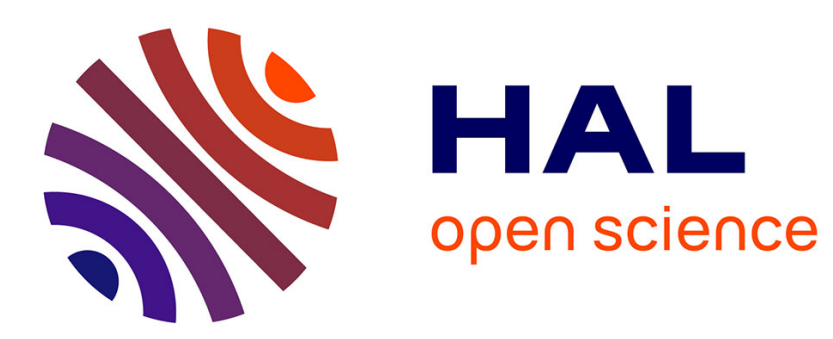

\title{
The mode branching route to localization of the finite-length floating elastica \\ Marco Rivetti, Sébastien Neukirch
}

\section{To cite this version:}

Marco Rivetti, Sébastien Neukirch. The mode branching route to localization of the finite-length floating elastica. 2013. hal-00904583

\section{HAL Id: hal-00904583 \\ https://hal.science/hal-00904583}

Preprint submitted on 14 Nov 2013

HAL is a multi-disciplinary open access archive for the deposit and dissemination of scientific research documents, whether they are published or not. The documents may come from teaching and research institutions in France or abroad, or from public or private research centers.
L'archive ouverte pluridisciplinaire HAL, est destinée au dépôt et à la diffusion de documents scientifiques de niveau recherche, publiés ou non, émanant des établissements d'enseignement et de recherche français ou étrangers, des laboratoires publics ou privés. 


\title{
The mode branching route to localization of the finite-length floating elastica
}

\author{
Marco Rivetti ${ }^{a} 1$ and Sébastien Neukirch ${ }^{b, c}$ \\ ${ }^{a}$ Max Planck Institute for Dynamics and Self-Organization (MPIDS), D-37073 Göttingen, Germany \\ ${ }^{b}$ CNRS, UMR 7190, Institut Jean Le Rond d'Alembert, F-75005 Paris, France \\ ${ }^{c}$ UPMC Univ Paris 06, UMR 7190, Institut Jean Le Rond d'Alembert, F-75005 Paris, France
}

\begin{abstract}
The beam on elastic foundation is a general model used in physical, biological, and technological problems to study delamination, wrinkling, or pattern formation. Recent focus has been given to the buckling of beams deposited on liquid baths, and in the regime where the beam is soft compared to hydrostatic forces the wrinkling pattern observed at buckling has been shown to lead to localization of the deformation when the confinement is increased. Here we perform a global study of the general case where the intensity of the liquid foundation and the confinement are both varied. We compute equilibrium and stability of the solutions and unravel secondary bifurcations that play a major role in the route to localization. Moreover we classify the post-buckling solutions and shed light on the mechanism leading to localization. Finally, using an asymptotic technique imported form fluid mechanics, we derive an approximated analytical solution to the problem.
\end{abstract}

\section{Introduction}

If submitted to a large enough axial load a slender elastic beam experiences a buckling instability, as first described by Euler more than two centuries ago. The length $L$ of the beam determines both the buckling threshold (the critical force scales as $L^{-2}$ ) and the shape of the buckling mode (the lateral deflection scales with $L$ ). Whenever the beam rests on a compliant substrate, buckling involves a competition between the bending of the beam and the deformation of the foundation. The former tends to select large wavelengths for the buckling mode, as in Euler problem, while the latter tends to select small wavelengths. This leads to the definition of another characteristic length-scale in the problem $\lambda \sim(B / K)^{1 / 4}$, with $B$ the bending stiffness of the beam and $K$ the rigidity of the foundation [1, 2. If $L \gg \lambda$ this new length-scale determines both the threshold for the instability, which now scales as $\lambda^{-2}$, and the shape of the buckling mode. In the post-buckling regime the shape of the buckled beam is first sinusoidal, as predicted by the linear stability analysis, but then a rich scenario opens as nonlinearities come into play.

The paradigm of the beam resting on an elastic foundation has been used to model and investigate many phenomena. Previous studies include for example the appearance of wrinkles in the human skin [2, 3, large folds or creases in membranes [4, 5, or delamination of thin films [6, 7. Problems arising in very different physical contexts and with length scales going from microscopic (Langmuir monolayers 8, lipid membranes 9], bacterial pellicles [10, hydrogels [11]) to macroscopic (foams [12, metallic thin films [13, particle rafts on a liquid [14]) seem to be amenable to models involving beams on elastic foundations. Consequently understanding and predicting the post-buckling behavior of such beams and membranes could have a wide impact on physics and biology, together with technological applications. For example fabrication of micro-lens and stretchable electronics based on wrinkling and creasing have recently been proposed 15, 16. The variety of post-buckling responses depends on the mechanical properties of both the beam and the foundation [2, 17. Many references exist on this classical problem of structural mechanics, see e.g. the works by Potier-Ferry [18] or Hunt et al. [19] in which the dynamical phase space analogy has proven most useful [20]. Recent studies on thin strips buckling either above elastomer [21] or liquid [4] foundations clearly show that, depending on the substrate, different evolutions take place. In the first case the sinusoidal buckling pattern has been shown to exhibit secondary, period doubling, bifurcations eventually leading to spatial chaos, while in the case of a liquid substrate the sinusoidal buckling pattern has been shown to localize into a well-defined fold, see [22] for a review. This manifestation of a localized buckling pattern has been explained in the case of an infinitely long beam $(L \rightarrow \infty)[23]$ where the shape of the beam is associated to an homoclinic orbit in the corresponding phase space [24]. In this homoclinic case, closed-form solutions have been found [25, 26, 27].

\footnotetext{
${ }^{1}$ email: marco.rivetti@ds.mpg.de
} 


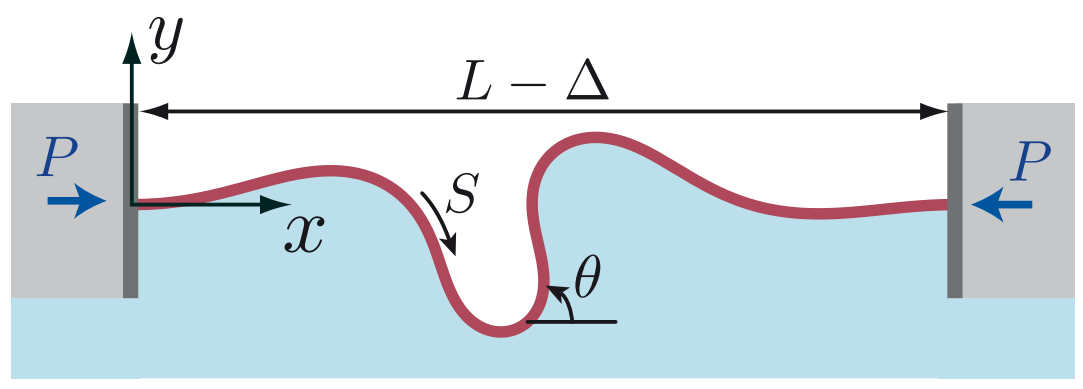

Figure 1: An elastic beam buckling on a liquid foundation under the action of an external force $P$. The beam is clamped at both ends. The deformation of the beam is described by the angle $\theta(S)$ between the tangent to the beam and the $x$-axis, where $S \in[0, L]$ is the arc-length along the beam.

In this paper we focus on the finite-length case of a beam on a liquid foundation and we show that, as for other elastic beams problems [28, the response of the finite-length elastic structure is different and more complex than that with infinite lengths. We describe both the buckling instability and the post-buckling regime, and show the existence of secondary bifurcations. These secondary bifurcations induce shape changes in the system as it deforms to a fold, giving it a mode branching route to localization.

The paper is organized as follows. In section 2 we present the problem and derive equilibrium equations. In section 3 we perform a linear stability analysis of the straight beam and predict the buckling threshold and modes. In section 4 we describe the non-linear response of the system in terms of equilibrium solutions and their stability, and we show that the system exhibits mode branching. In section 5 we perform an asymptotic expansion analysis and give an analytical approximated solution.

\section{The floating elastica problem}

We consider an elastic beam resting on a liquid surface and we study its equilibrium configurations under the action of a compressive load. As indicated in Fig. 1, we consider planar deformations of the beam, $x$ and $y$ denoting the horizontal and vertical directions respectively. The density of the liquid is noted $\rho$ and the acceleration of gravity $g$. The beam has length $L$, width $w$, thickness $e$, and density $\rho_{s}$. We work under the slender $(L \gg w \sim e)$ Euler-Bernoulli hypotheses where the beam is considered inextensible and unshearable. Configurations are thus fully described by the position and orientation of the centerline. We use the arc length $S \in(0, L)$ and note $\theta(S)$ the angle between the tangent of the beam and the horizontal. Force and torque balance for the internal force $\mathbf{N}(S)=N_{x}(S) \mathbf{e}_{\mathbf{x}}+N_{y}(S) \mathbf{e}_{\mathbf{y}}$ and the internal moment $\mathbf{M}(S)=M(S) \mathbf{e}_{\mathbf{z}}$ yield

$$
\begin{aligned}
& N_{x}^{\prime}(S)=+f(S) \sin \theta(S) \\
& N_{y}^{\prime}(S)=-f(S) \cos \theta(S) \\
& M^{\prime}(S)=N_{x}(S) \sin \theta(S)-N_{y}(S) \cos \theta(S)
\end{aligned}
$$

These equations are written per unit width. Here, ()$^{\prime}=\mathrm{d}() / \mathrm{d} S$ denotes the derivative with respect to the arc-length $S$ and $f(S)$ is the distributed force along the beam. As we neglect the weight of the floating beam, $f(S)$ is simply given by the hydrostatic pressure in the liquid:

$$
f(S)=-\rho g Y(S)
$$


Kinematics and bending constitutive relation yield three supplementary equations between the position $(X(S), Y(S))$ of the beam, its orientation $\theta(S)$, and the bending moment $M(S)$ :

$$
\begin{aligned}
X^{\prime}(S) & =\cos \theta(S) \\
Y^{\prime}(S) & =\sin \theta(S) \\
B \theta^{\prime}(S) & =M(S),
\end{aligned}
$$

where $B=E e^{3} w / 12$ is the bending stiffness, $E$ being the Young modulus. Buckling is the consequence of the action of the horizontal compressive load $P=N_{x}(L)=-N_{x}(0)$, and we monitor the post-buckled configurations with the end displacement $\Delta=L-[X(L)-X(0)]$. Introducing dimensionless quantities

$$
s=\frac{S}{L} ; x=\frac{X}{L} ; y=\frac{Y}{L} ; \delta=\frac{\Delta}{L} ; n=\frac{N L^{2}}{B} ; p=\frac{P L^{2}}{B} ; m=\frac{M L}{B}
$$

we rewrite the full set of equilibrium equations (1)-(3) as

$$
\begin{array}{rll}
n_{x}^{\prime}(s)=-\eta^{4} y \sin \theta & , \quad n_{y}^{\prime}(s)=+\eta^{4} y \cos \theta(s) \\
m^{\prime}(s)=n_{x} \sin \theta-n_{y} \cos \theta & , \quad \theta^{\prime}(s)=m \\
x^{\prime}(s)=\cos \theta & , \quad y^{\prime}(s)=\sin \theta
\end{array}
$$

where we have introduced the dimensionless parameter $\eta=L / L_{\mathrm{eh}}$. The elasto-hydrostatic length $L_{\mathrm{eh}}=(B / \rho g w)^{1 / 4}$ compares the bending stiffness of the beam to the weight of the liquid [4, 29]. Here $\eta$ is a measure of the intensity of the liquid foundation on which the beam rests. The case $\eta=0$ corresponds to classical Euler buckling [30] while the case $\eta \rightarrow \infty$ corresponds to either an infinitely long beam or a infinitely heavy liquid, and has been studied in [23, 25, 26, 27]. In this case, the solution is called homoclinic since in the phase space of Eq. (7) its trajectory describes an homoclinic connection to the origin.

We consider rigid boundary conditions where the beam position and orientation are prescribed at both extremities:

$$
\begin{gathered}
x(0)=0 ; y(0)=0 ; \theta(0)=0 \\
x(1)=1-\delta ; y(1)=0 ; \theta(1)=0 .
\end{gathered}
$$

We will compute equilibrium and stability of the system for increasing values of the imposed horizontal displacement $\delta$. We remark that it is possible to recast the system of equations (5) into one equation for the variable $\theta(s)$ [25]:

$$
\theta^{\prime \prime \prime \prime}(s)+\frac{3}{2} \theta^{\prime \prime}(s) \theta^{\prime}(s)^{2}-\mathcal{H} \theta^{\prime \prime}(s)+\eta^{4} \sin \theta(s)=0
$$

where $\mathcal{H}=\frac{1}{2} m^{2}(s)+n_{x}(s) \cos \theta(s)+n_{y}(s) \sin \theta(s)$ is the Hamiltonian, a conserved quantity along the beam: $d \mathcal{H} / d s \equiv$ 0 . Its value is then evaluated at, for example, $s=0: \mathcal{H}=\frac{1}{2} \theta^{\prime 2}(0)-p$.

\section{Buckling threshold and modes}

\subsection{Linear analysis and buckling threshold}

For small deflections, equation $(7)$ is linearized to

$$
\theta^{\prime \prime \prime \prime}+p \theta^{\prime \prime}+\eta^{4} \theta=0
$$

which is equivalent to:

$$
y^{\prime \prime \prime \prime}+p y^{\prime \prime}+\eta^{4} y=0 .
$$

We seek for solutions in the form $y \propto \exp (i k s)$. The characteristic equation is:

$$
k^{4}-p k^{2}+\eta^{4}=0
$$




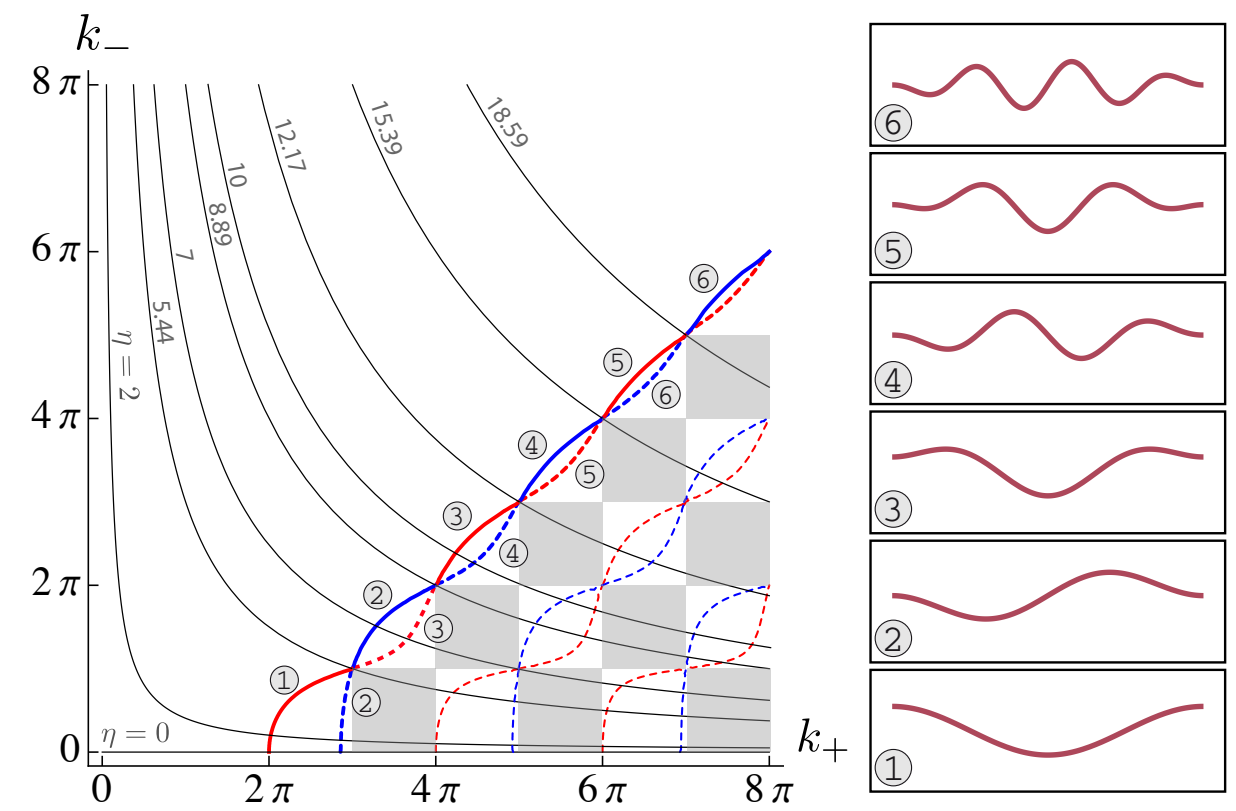

Figure 2: Buckling wavelengths and modes for the floating elastica. Red and blue curves are solutions of equation 15 and correspond to symmetric and anti-symmetric buckling modes, respectively. The mode selected at buckling belong to the continuous thick line (partly red, partly blue). The hyperboles $k_{-}=\eta^{2} / k_{+}$are also shown for several value of $\eta$. Shaded regions correspond to forbidden value of $k_{-}$and $k_{+}$. Mode shapes are given on the right, together with the label $i$ corresponding to the number of zeros of the function $\theta(s)$ for $0<s<1$.

and has four solutions: $k= \pm k_{+}$and $k= \pm k_{-}$, with

$$
k_{+}=\sqrt{p / 2+\sqrt{(p / 2)^{2}-\eta^{4}}} \quad ; \quad k_{-}=\sqrt{p / 2-\sqrt{(p / 2)^{2}-\eta^{4}}} .
$$

We note that $k_{+} k_{-}=\eta^{2}$ and $k_{+}^{2}+k_{-}^{2}=p$. The buckling mode of the beam takes the form

$$
y(s)=A \sin k_{+} s+B \cos k_{+} s+C \sin k_{-} s+D \cos k_{-} s
$$

where the coefficients $A, B, C$ and $D$ are specified via the boundary conditions. At the left end, clamped boundary conditions $y(0)=y^{\prime}(0)=0$ simply give $D=-B$ and $C=-A k_{+} / k_{-}$, while at the right end boundary conditions $y(1)=y^{\prime}(1)=0$ lead to an homogeneous system for $A$ and $B$ :

$$
\left(\begin{array}{ll}
\alpha_{11} & \alpha_{12} \\
\alpha_{21} & \alpha_{22}
\end{array}\right)\left(\begin{array}{l}
A \\
B
\end{array}\right)=\left(\begin{array}{l}
0 \\
0
\end{array}\right)
$$

with:

$$
\begin{gathered}
\alpha_{11}=k_{-} \sin k_{+}-k_{+} \sin k_{-} \quad ; \quad \alpha_{12}=k_{-} \cos k_{+}-k_{-} \cos k_{-} \\
\alpha_{21}=k_{+} \cos k_{+}-k_{+} \cos k_{+} \quad ; \quad \alpha_{22}=-k_{+} \sin k_{+}+k_{-} \sin k_{-} .
\end{gathered}
$$

As usual in buckling analysis, the condition for system 13 to have a non-zero solution is that the determinant $\alpha_{11} \alpha_{22}-\alpha_{12} \alpha_{21}$ takes the zero value. This gives a relation between the critical values of $k_{+}$and $k_{-}$:

$$
2 k_{+} k_{-}\left(\cos k_{+} \cos k_{-}-1\right)+\left(k_{+}^{2}+k_{-}^{2}\right) \sin k_{+} \sin k_{-}=0 .
$$

Solutions of this equation is a discrete family of curves in the $\left(k_{+}, k_{-}\right)$plane, see Fig. 2, As we consider positive values of $k_{+}$and $k_{-}$equation (15) implies that $\sin k_{+} \sin k_{-} \geq 0$, which forbid the shaded regions in Fig. 2. Taking (11) into 
account, 15 is eventually an equation for the buckling threshold $p$ as a function of $\eta$. The case $\eta=0$ corresponds to the planar elastica, where $k_{-}=0$. The mode selected at buckling is then $k_{+}=2 \pi$ and $p=4 \pi^{2}$. For $\eta>0$, approximate expressions for this mode can be found:

$$
\begin{aligned}
& k_{-}=\pi[f(\eta)-1]+(1 / 2) \arccos \left[1-\frac{1-\cos (2 \pi f(\eta))}{f^{2}(\eta)}\right] \\
& k_{+}=2 \pi+\pi[f(\eta)-1]-(1 / 2) \arccos \left[1-\frac{1-\cos (2 \pi f(\eta))}{f^{2}(\eta)}\right]
\end{aligned}
$$

with $f(\eta)=\sqrt{1+(\eta / \pi)^{2}}$. This corresponds to the left-most curve in Fig. 2. As $\eta \gg 1$, the buckling threshold is approximated by $p=4 \pi^{2}+2 \eta^{2}$, and as $\eta \rightarrow \infty$, we have $p \rightarrow 2 \eta^{2}$. Introducing the rescaled load $\bar{p}=p / \eta^{2}$ we recover the result $\bar{p}=2$ for an infinite long beam [23, 25].

\subsection{Buckling modes}

Once the coefficients $A, B, C$ and $D$ are specified, the buckling mode writes:

$$
y(s)=\left(k_{-} \sin k_{+}-k_{+} \sin k_{-}\right)\left(\cos k_{+} s-\cos k_{-} s\right)-\left(\cos k_{+}-\cos k_{-}\right)\left(k_{-} \sin k_{+} s-k_{+} \sin k_{-} s\right),
$$

and we see it is a quasiperiodic function with two wavelengths $2 \pi / k_{+}$and $2 \pi / k_{-}$. In order to analyze which mode is selected at buckling, we show in Fig. 2 the zeros of Eq. 15 in the $\left(k_{+}, k_{-}\right)$plane. Red curves represent symmetric buckling modes and blue curves represent antisymmetric buckling modes. By symmetric (respectively antisymmetric) we mean that the function $y(\sigma=s-1 / 2)$ is even (resp. odd) with regard to $\sigma$. We also show the curves corresponding to constant values of $\eta$ : these curves are the hyperboles $k_{-}=\eta^{2} / k_{+}$. For any given value of $\eta$, a discrete family of buckling modes exists, corresponding to the points at which the hyperbole intersects red and blue curves. Among all these modes, the mode selected at buckling is situated on the left-most curve in the figure, designated with a continuous thick line. All the other curves correspond to unstable modes and are plotted with dashed lines. Note that the modes corresponding to $\eta=0$ (Euler buckling) are situated along the degenerated hyperbole $k_{-}=0$ (the horizontal axis). Only in this special case the buckling mode is a single-periodic function, with wavelength $2 \pi / k_{+}=1$.

We now focus our attention on the first pair of curves (plotted with thick lines in Fig. 2p). It appears that these two curves continuously cross each other, at points where $k_{ \pm}$are multiple of $\pi$. The mode selected at buckling always belongs to one of these two curves and may be symmetric or antisymmetric, depending on the value of $\eta$. For instance, it is symmetric for $\eta<5.44$ and becomes antisymmetric as $\eta>5.44$.

Finally, if we label the modes with the number $i$ of zeros of the function $\theta(s)$ for $0<s<1$, we have that at each switching point the mode which becomes unstable converts itself from a $n$-mode to a $(n+2)$-mode. For instance, at $\eta=7$ the mode selected at buckling is an $i=2$ antisymmetric mode, while the second (unstable) mode is a $i=3$ symmetric mode. At $\eta=10$, the mode selected at buckling is a $i=3$ symmetric mode and the second mode is an $i=4$ antisymmetric mode. Note that modes belonging to the other pairs of curves in Fig. 2 always have higher $i$ labels than those on the first pair, and are always unstable.

\subsection{Switching points}

We call switching points the intersections of the first pair of curves in Fig. 2. These points are such that $k_{-}=n \pi$ and $k^{+}=(n+2) \pi$, with $n>0$ being an integer. From 10 and 11 we have $\eta=\pi\left(n^{2}+2 n\right)^{1 / 2}$ and $p=2 \pi^{2}\left(n^{2}+2 n+2\right)$. For example the first switching point, $n=1$, has $\eta=\sqrt{3} \pi \simeq 5.44$ and $p=10 \pi^{2}$. Values for the first six switching points are given in Fig. 2. Since at any switching point $k_{+}$and $k_{-}$are multiples of $\pi$, all the $\alpha$ coefficients appearing in Eq. (13) are zero. Therefore the boundary conditions at $s=1$ are automatically satisfied and there is no condition on the coefficients $A$ and $B$, which can be chosen arbitrarily. Consequently at each switching point the selected mode at buckling is not unique and there is rather a continuous family of modes. Symmetric, antisymmetric, and non-symmetric solutions belong to this family, and it is not possible to determine a priori which one is selected. 
(a)

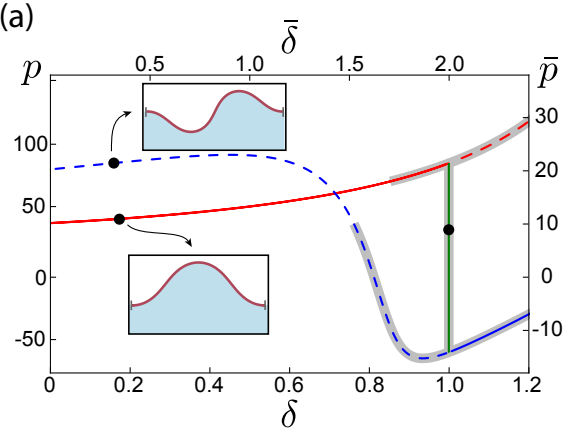

(d)

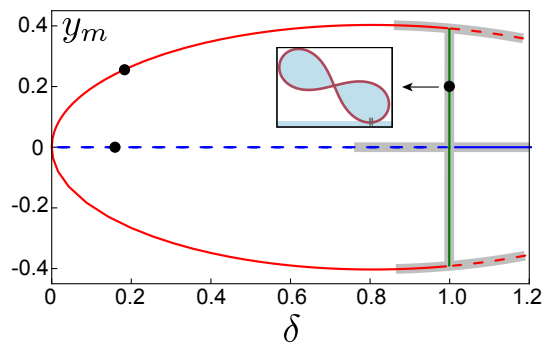

(b)

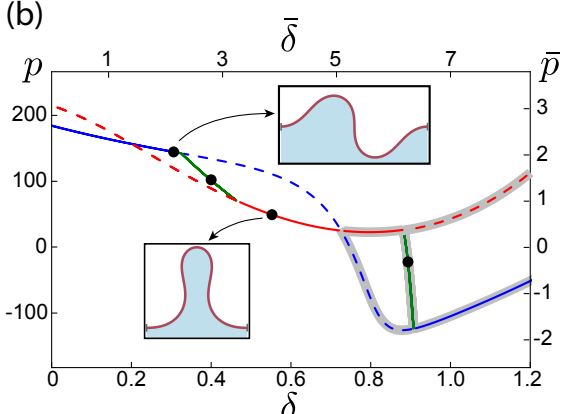

(e)

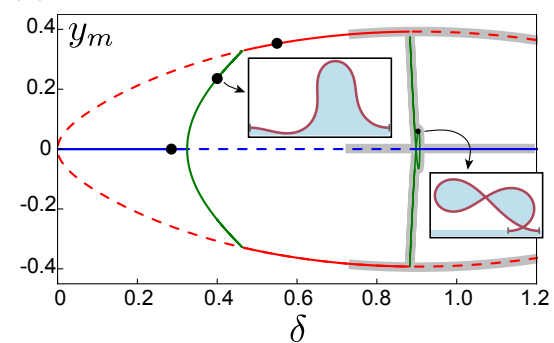

(c)

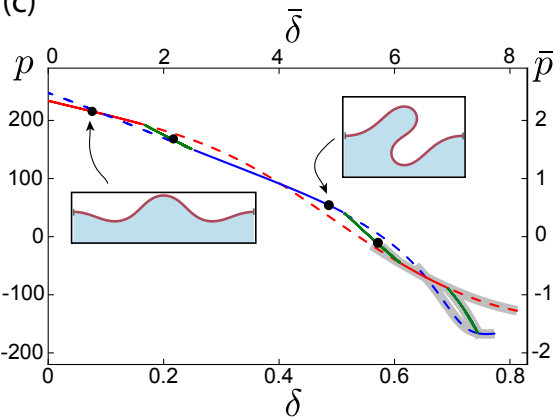

(f)

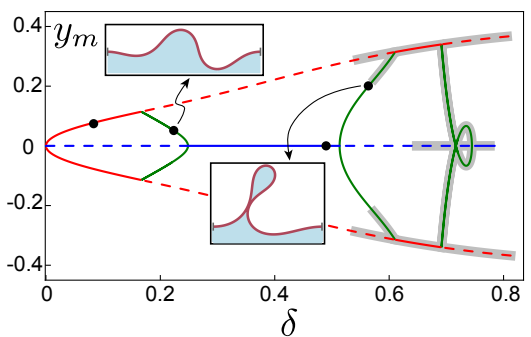

Figure 3: Post-buckling paths for $(\mathrm{a})(\mathrm{d}) \eta=2$, (b)(e) $\eta=7$, and (c)(f) $\eta=10$. The compression $p$ and vertical deflection $y_{m}=y(s=1 / 2)$ are plotted as function of the imposed displacement $\delta$. Scaled variables $\bar{p}=p / \eta^{2}$ and $\bar{\delta}=\delta \eta$ are used for comparison with the infinite length case. Continuous (respectively dashed) curves correspond to stable (resp. unstable) solutions. Red (respectively blue) curves correspond to symmetric (resp. anti-symmetric) solutions. Green curves correspond to connection paths, where solutions have no symmetry. Shaded parts of the paths correspond to solutions where self-crossing occurs. 

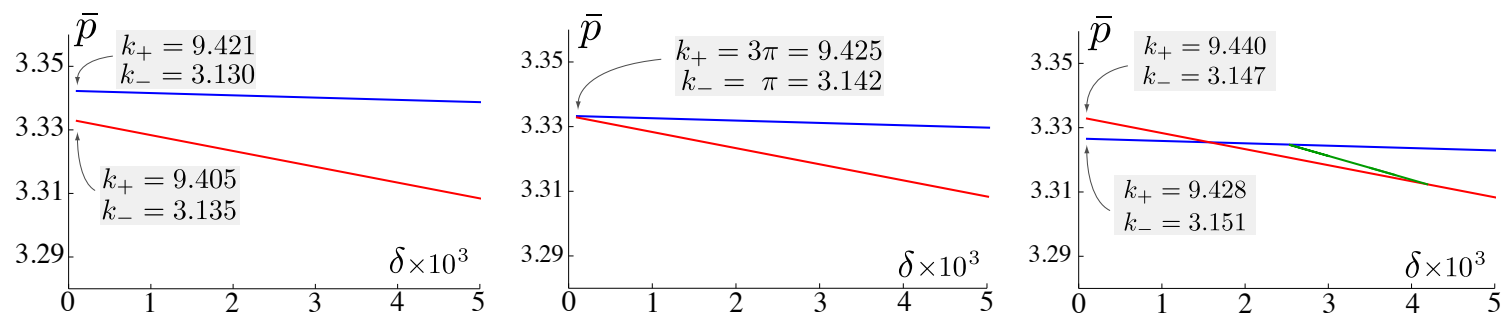

Figure 4: Post-buckling paths for $\eta=5.43$ (left), $\eta=\pi \sqrt{3}$ (middle), and $\eta=5.45$ (right). A connection branch appears at small values of $\delta$ as the buckling modes switch.

\section{Nonlinear post-buckling analysis}

\subsection{Equilibrium solutions}

We now analyze the post-buckling regime by numerically solving the non-linear system of equilibrium equations (5)(6). Results are obtained using a continuation algorithm, starting from points at the buckling threshold and following the equilibrium into the post-buckling domain. We plot in Fig. 3 the continuation branches for three values $\eta=2,7$ and 10. For $\eta=2$ (see Fig. 3 a and d), the antisymmetric mode bifurcates at $p=81.1(\bar{p}=20.3)$, but the symmetric mode buckles for a lower compression $p=40.7(\bar{p}=10.2)$ and is therefore selected. As the displacement $\delta$ increases, these two modes have separated evolutions until $\delta \simeq 1$. Here, a new branch appears and connects the symmetric to the antisymmetric mode. Along this branch, the solutions are non-symmetric (see inset in Fig. 3 d), and stable. Note that this connection already exists for $\eta=0$ in the planar elastica case [31]. For $\eta=7$ (see Fig. 3 b b and e) symmetrical and anti-symmetrical modes both bifurcates sub-critically. Moreover, the anti-symmetrical mode is now the selected mode at buckling $(p=128.9, \bar{p}=2.6)$. Two connections exist between the symmetric and antisymmetric branches, for $\delta \sim 0.4$ and the other for $\delta \sim 0.9$. Although the second one $(\delta \sim 0.9)$ may just be seen as an evolution of the connection already present for $\eta=2$, the first one $(\delta \sim 0.4)$ is new. Here also the solutions along the connections are non-symmetric (see inset in Fig. 3 e), and stable. For $\eta=10$, buckling modes are reversed again, the symmetrical being selected. A third connection now exists between the principal branches (see Fig. $3 \mathrm{c}$ and $\mathrm{f}$ ) made of stable, non-symmetrical solutions. Note that in all this analysis we have pushed the continuation beyond the self-contact of the beam, thus exploring non-physical configurations. In Fig. 3 regions corresponding to self-crossing configurations appear shaded.

In Fig. 4 we illustrate the birth of a connection as $\eta$ crosses $\pi \sqrt{3}$, precisely when the first switching of buckling mode occurs. In Fig. 4 left (for $\eta \lesssim \pi \sqrt{3}$ ) there is no secondary bifurcation along the two equilibrium paths, whereas in Fig. 4 right (for $\eta>\pi \sqrt{3}$ ) a connection branch clearly appears for small values of $\delta$. This connection branch is born at $\eta=\pi \sqrt{3}$ (Fig. 4. middle) as the two buckling modes switch. In general, we observe that for each inversion of the buckling modes, a new connection branch appears in the post-buckling domain. Apart from the first one, already present in the planar elastica case, these connections are then directly related to switching points. More precisely, the number of connection branches is given by the largest integer $n$ such that $\pi\left(n^{2}-1\right)^{1 / 2} \leq \eta$. Connections between branches are an example of secondary bifurcation. This phenomenon has already been reported in the case of buckling on elastic foundations, and it has been demonstrated that the origin of these secondary bifurcations is the switching point at the buckling itself [32, 33, 34]. As $\eta$ becomes larger more connections appear and each of them spans over a small $\delta$ interval. Eventually, as $\eta \rightarrow \infty$, an infinite number of connections exists, each for a precise $\delta$ value. In this limit, the symmetric and anti-symmetric paths are continuously connected, as reported in [26, 27.

\subsection{Stability of the solutions}

Due to the presence of secondary bifurcations in the post-buckling domain, a careful analysis of the stability of the solutions is needed in order to determine which path(s) is (are) followed as the displacement $\delta$ is increased. The stability analysis consists in computing the small amplitude vibrations of the system around its equilibrium solution. 


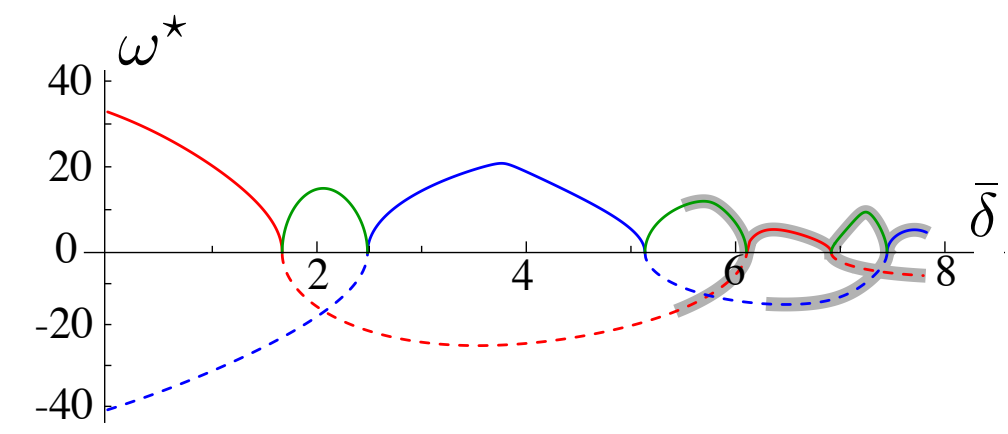

Figure 5: Lowest frequency for each of the equilibrium paths for $\eta=10$ (see Fig. 3 c and f). A positive (respectively negative) $\omega^{\star}$ corresponds to a stable (resp. unstable) equilibrium solution. Red (respecticely blue) curves correspond to symmetric (resp. anti-symmetric) solutions. Green curves correspond to connection paths, where solutions have no symmetry. Shaded parts of the paths correspond to solutions where self-crossing occurs. Note that solutions on the connection paths are always stable.

We first recall non-dimensional dynamic Kirchhoff equations:

$$
\begin{aligned}
x^{\prime}(s, t) & =\cos \theta(s, t) \\
y^{\prime}(s, t) & =\sin \theta(s, t) \\
\theta^{\prime}(s, t) & =m(s, t) \\
m^{\prime}(s, t) & =n_{x}(s, t) \sin \theta(s, t)-n_{y}(s, t) \cos \theta(s, t)+\beta \ddot{\theta}(s, t) \\
n_{x}^{\prime}(s, t) & =-\eta^{4} y(s, t) \sin \theta(s, t)+\ddot{x}(s, t) \\
n_{y}^{\prime}(s, t) & =\eta^{4} y(s, t) \cos \theta(s, t)+\ddot{y}(s, t)
\end{aligned}
$$

where ()$^{\prime}=\mathrm{d} / \mathrm{d} s$ is the derivative with respect to the arc-length $s$ and $\dot{()}=\mathrm{d} / \mathrm{d} t$ is the derivative with respect to time $t$. The physical time $T$ has been rescaled as $t=T / \sqrt{\rho_{s} e L^{4} / B}$, where $\rho_{s}$ is the density of the beam. The parameter $\beta=e^{2} /\left(12 L^{2}\right)$ is the slenderness ratio of the beam, and since $\beta \ll 1$ we neglect the term $\beta \ddot{\theta}(s, t)$ in the following. We look at the evolution of a small perturbations around the equilibrium using the decomposition

$$
a(s, t)=a_{e}(s)+\epsilon \hat{a}(s) \exp (i \omega t)
$$

for all the variables in $(19)$, i.e. $a=x, y, \ldots, n_{y}$. The quantity $a_{e}(s)$ stands for the equilibrium solution and $\hat{a}(s)$ is the linear vibration mode around the (nonlinear) equilibrium. Injecting (20) in (19), making use of (5), and keeping only the first order in $\epsilon$ yields the system of equations for the vibration modes

$$
\begin{aligned}
\hat{x}^{\prime} & =-\hat{\theta} \sin \theta_{e} \\
\hat{y}^{\prime} & =\hat{\theta} \cos \theta_{e} \\
\hat{\theta}^{\prime} & =\hat{m} \\
\hat{m}^{\prime} & =n_{x, e} \cos \hat{\theta}+\hat{n}_{x} \sin \theta_{e}+n_{y, e} \sin \hat{\theta}-\hat{n}_{y} \cos \theta_{e} \\
\hat{n}_{x}^{\prime} & =-\eta^{4}\left(\hat{y} \sin \theta_{e}+y_{e} \cos \theta_{e} \hat{\theta}\right)-\omega^{2} \hat{x} \\
\hat{n}_{y}^{\prime} & =-\eta^{4}\left(\hat{y} \cos \theta_{e}-y_{e} \sin \theta_{e} \hat{\theta}\right)-\omega^{2} \hat{y}
\end{aligned}
$$

These equations have to be solved with the boundary conditions:

$$
\hat{\theta}(0)=\hat{y}(0)=\hat{x}(0)=\hat{\theta}(1)=\hat{y}(1)=\hat{x}(1)=0 .
$$



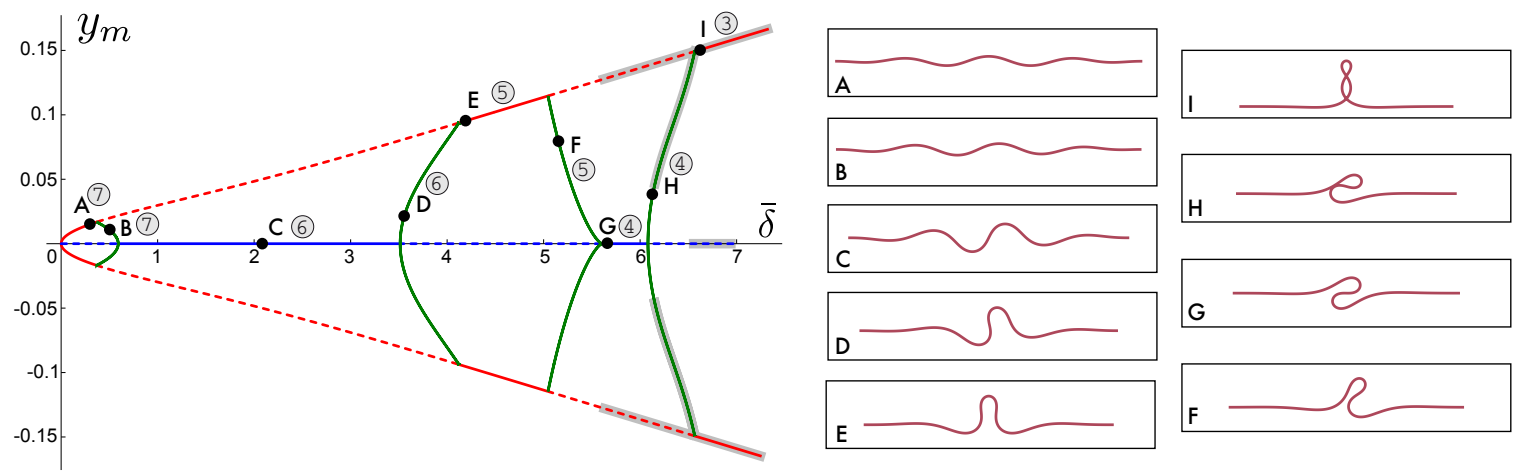

Figure 6: Route to localization for $\eta=22$. The post-buckling equilibrium shapes are labeled with the integer $i$, corresponding to the number of points along the beam where the angle $\theta(s)$ is zero, which decreases by one unit each time the solutions leaves a non-symmetrical branch. The shapes exhibit less and less oscillations and the deformation localizes to a narrow region of the beam.

System 21 can be seen as a generalized eigenvalue problem with eigenvalues $\omega^{2}$. For each equilibrium solution, along each bifurcation path, we compute the lowest eigenvalues $\omega^{2}$. The equilibrium solution is said to be stable if $\omega^{2}>0$ and unstable if $\omega^{2}<0$. In Fig. 3 plain (respectively dotted) curves correspond to paths of stable (resp. unstable) solutions. In Fig. 5 we show $\omega^{\star}=\operatorname{sign}\left(\omega^{2}\right) \sqrt{\left|\omega^{2}\right|}$ as a function of $\bar{\delta}$ for the case $\eta=10$. Stability results announced in Section 4.1 (see Fig. 3 c-f) are here clearly demonstrated: at buckling the symmetric solution (red curve) is stable (positive $\omega^{\star}$ ) and the anti-symmetric one (blue curve) is unstable (negative $\omega^{\star}$ ). As $\bar{\delta}$ is increased and reaches $\bar{\delta} \simeq 1.7$, the symmetric solution becomes unstable. The stable solution is now the non-symmetric one (green curve). At $\bar{\delta} \simeq 2.5$ another bifurcation arrises and the stable solution is now the anti-symmetric one. The next bifurcation at $\bar{\delta} \simeq 5.1$ sees the non-symmetric solution becoming stable again. This solution eventually hands over the baton to symmetric solution at $\bar{\delta} \simeq 6.1$, and so on. This scenario of alternating stability between symmetric and anti-symmetric solutions with non-symmetric solutions in between is a general feature and exists for all $\eta$. Note that connection branches of non symmetric solutions are always stable.

We finally remark that we do not consider the inertia of the liquid. The computed frequencies are then not accurate and we do not detect instabilities arising from the liquid phase. Nevertheless in the regime studied here we believe the stability of the system is correctly predicted.

\subsection{Mode branching and localization}

In the infinite length (or infinitely heavy liquid) case, it has been shown that as the axial displacement $\delta$ is increased the deformation of the beam becomes localized into a narrow region [4, 23, 25]. In the present finite-length case, localization also happen and we discuss here the route from buckling to localized solutions. The connection branches, which link the symmetric and antisymmetric branches, are always stable. This phenomenon has been called mode branching and is typically observed with stiffening elastic foundations [34. Here mode branching lies at the heart of the localization process as it is through the connecting branches that the deformation localizes into a fold. This is true in particular for large values of $\eta$, where many secondary bifurcations (and hence connection branches) arises. To illustrate this, we display in Fig. 6 the bifurcation paths for $\eta=22$ together with several beam shapes for increasing values of the displacement $\delta$. Qualitatively, we observe that the smooth sinusoidal pattern (configuration A) first localizes into a well defined symmetric fold (configuration E), then into an anti-symmetric fold (configuration G), and eventually to self-crossing solutions (configuration I). The non-symmetric shapes (configurations B, D, F and H) involved in the localization process are also represented.

As we did for the buckling modes, we label the post-buckling configurations with an integer $i$ equal to the number of points for which $\theta(s)=0$ for $0<s<1$. The label of the equilibrium solutions, also displayed in Fig. 6, decreases as the displacement $\delta$ increases. More precisely the label decreases by one unit each time the solution leaves a nonsymmetric branch. The reason for this lies in the fact that the ending configuration of every connection branch has the 


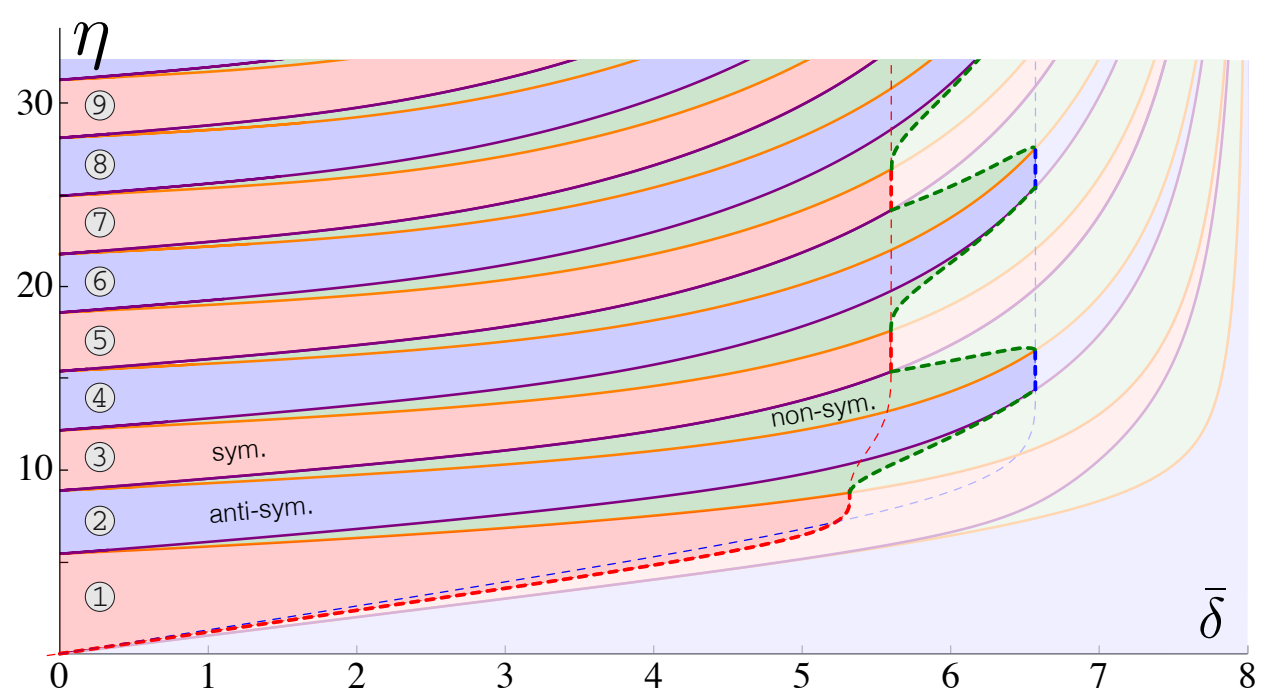

Figure 7: Phase diagram for the floating elastica. Depending on the displacement $\bar{\delta}$ and the strength $\eta$ of the liquid foundation the stable configuration of the system might be symmetrical (red regions), anti-symmetrical (blue regions), or non-symmetrical (green regions). The label $i$, corresponding to the number of points along the beam where the angle $\theta(s)$ is zero, is also given. Dashed curves correspond to the moment where the beam first self-contacts, with configurations with higher $\bar{\delta}$ exhibiting self-crossing.

special characteristic that the internal moment vanishes at the extremities of the beam $m(0)=m(1)=0$. Recalling that $m(s)=\theta^{\prime}(s)$, we see that for this ending configuration the function $\theta(s)$ has a double root at $s=0$ and $s=1$. For the following configuration (on the next symmetrical or anti-symmetrical branch) the function $\theta(s)$ will lose one root, that is the label $i$ will decrease by one unit. In a parallel manner the starting configuration of every connection branch has the special characteristic that the vertical component of the internal force vanishes at the extremities of the beam: $n_{y}(0)=n_{y}(1)=0$. We refer to these starting and ending configurations as $N$ and $M$ points respectively, and we infer from the equilibrium equations (5) that for $M$ points all the odd derivatives of $\theta(s)$ vanish at both ends, while for $N$ points all the even derivatives of $\theta(s)$ vanish at both ends.

Finally we summarize the post-buckling response of the beam in a phase diagram plotted in the plane $(\bar{\delta}, \eta)$, see Fig. 7. In each region of the plane, the stable configuration is given, together with its label $i$. The regions are separated by $M$ and $N$ curves, and on the vertical $\bar{\delta}=0$ axis the $M$ and $N$ curves emerge two by two from the switching points described in Section 3 . The limit where self-contact first occurs is also drawn.

\section{Approximate solution for large $\eta$}

\subsection{Successive complementary expansion method}

In the case of an infinitely long beam (or a infinitely heavy liquid), $\eta \rightarrow \infty$, the solution can be written in closed form [25, 26, 27]:

$$
\begin{aligned}
& \theta_{0}(s)=4 \arctan \left[\frac{c}{k} \frac{\sin \left(k \eta\left[s-\frac{1}{2}+\phi\right]\right)}{\cosh \left(c \eta\left[s-\frac{1}{2}\right]\right)}\right] \\
& y_{0}(s)=-\frac{4 c k}{\eta} \frac{k \cos \left(k \eta\left[s-\frac{1}{2}+\phi\right]\right) \cosh \left(c \eta\left[s-\frac{1}{2}\right]\right)+c \sin \left(k \eta\left[s-\frac{1}{2}+\phi\right]\right) \sinh \left(c \eta\left[s-\frac{1}{2}\right]\right)}{k^{2} \cosh ^{2}\left(c \eta\left[s-\frac{1}{2}\right]\right)+c^{2} \sin ^{2}\left(k \eta\left[s-\frac{1}{2}+\phi\right]\right)}
\end{aligned}
$$

where $c=(1 / 2) \sqrt{2-\bar{p}}, k=(1 / 2) \sqrt{2+\bar{p}}$, and $\bar{p}=p / \eta^{2}$. The parameter $\phi$ allows us to treat symmetric $(\phi=0)$ and anti-symmetric $(\phi=\pi /(2 k \eta))$ solutions with the same formula [26]. We call homoclinic this solution since $\theta_{0}(s)$ 

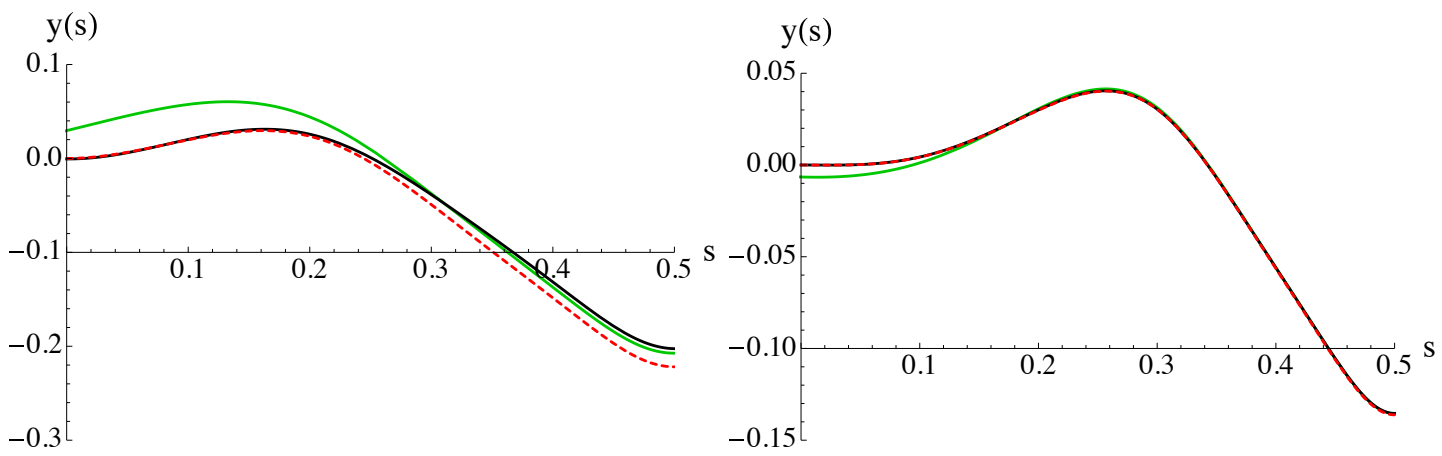

Figure 8: The deflection $y(s)$ for a symmetrical solution for the left half of the beam: comparison between $(i)$ the numerical resolution (red dashed curve), (ii) the homoclinic solution given by (23) (green curve), and (iii) the SCE approximation (24) (black curve), for $\bar{\delta}=4$ and $\eta=10$ (left), $\eta=15$ (right). The SCE approximation uniformly converges to the numerical solution as $\eta$ grows.

and its derivatives vanish as $s \rightarrow \pm \infty$. We note that even for moderate $\eta$ the homoclinic solution is not far from the numerical solution, except in two small regions near the boundaries $s=0$ and $s=1$, see Fig. 8. The mismatch stems from the fact that the homoclinic solution does not satisfy the boundary conditions. As $\eta$ increases we observe that the numerical solution becomes closer to the homoclinic solution, and that the size of the mismatch region decreases as $1 / \eta$. All the ingredients for a boundary layer approach are then present. Classical matched asymptotic expansions (MAE) would require to find approximated solutions for so-called outer and inner regions and to matche them in a intermediate region. Then an approximation valid in the entire domain would have to be found, this last step being non obvious (a lucky guess sometimes makes the trick). A powerful alternative is the so-called method of successive complementary expansions (SCE) [35], which we employ here. The two main advantages of the SCE method is that no matching is required and that a uniformly valid approximation is automatically obtained. For comparison purposes we solve the problem using the MAE method in A. Here we propose a solution of the form:

$$
\begin{aligned}
& \theta(s)=\theta_{0}(s)+\epsilon \theta_{1}(\tilde{s})+\ldots \\
& y(s)=y_{0}(s)+\epsilon^{2} y_{1}(\tilde{s})+\ldots
\end{aligned}
$$

where $s=\epsilon \tilde{s}$ and $\epsilon=1 / \eta$, and we inject it into the equilibrium equation 77). Writing $(\dot{)}=\mathrm{d}() / \mathrm{d} \tilde{s}$ and noting that $\dot{\theta_{1}}=\epsilon \theta_{1}^{\prime}$, we obtain

$$
\theta_{0}^{\prime \prime \prime \prime}+\frac{\dddot{\theta_{1}}}{\epsilon^{3}}+\frac{3}{2}\left(\theta_{0}^{\prime \prime}+\frac{\ddot{\theta_{1}}}{\epsilon}\right)\left(\theta_{0}^{\prime}+\dot{\theta_{1}}\right)^{2}-\mathcal{H}\left(\theta_{0}^{\prime \prime}+\frac{\ddot{\theta_{1}}}{\epsilon}\right)+\eta^{4} \sin \left(\theta_{0}+\epsilon \theta_{1}\right)=0
$$

with $\mathcal{H}=-p+\frac{1}{2}\left[\theta_{0}^{\prime}(0)+\dot{\theta_{1}}(0)\right]^{2}$. As $p \sim \eta^{2}$ (see Section 3, or [25]) and anticipating the scaling $\theta_{0}^{\prime}(0)+\dot{\theta_{1}}(0) \sim \mathrm{e}^{-\eta}$ (see Eq. 31), we write $\mathcal{H}=-p$. Recalling that $\theta_{0}(s)$ satisfies $\theta_{0}^{\prime \prime \prime \prime}+\frac{3}{2} \theta_{0}^{\prime \prime} \theta_{0}^{\prime 2}+p \theta_{0}^{\prime \prime}+\eta^{4} \sin \theta_{0}=0$, we find that the leading, $\epsilon^{-3}$, order of 25 is

$$
\dddot{\theta_{1}}+\bar{p} \ddot{\theta_{1}}+\theta_{1} \cos \theta_{0}=0
$$

where $\bar{p}=\epsilon^{2} p$. Note that the solution $\theta_{0}(s)$ depends on $\epsilon$. We nevertheless want an equation for $\theta_{1}$ that is independent of $\epsilon$ and we consequently set $\cos \theta_{0}=1$ in $26 \sqrt{2}$. We then look for a solution $\theta_{1}(\tilde{s})=\dot{y}_{1}(\tilde{s})$ with

$$
y_{1}(\tilde{s})=\alpha \sinh (c \tilde{s}) \sin (k \tilde{s})+\beta \sinh (c \tilde{s}) \cos (k \tilde{s})+\gamma \cosh (c \tilde{s}) \sin (k \tilde{s})+\xi \cosh (c \tilde{s}) \cos (k \tilde{s})
$$

Two of the unknown coefficients $(\alpha, \beta, \gamma, \xi)$ are found using the boundary conditions at the left end, $y(0)=\theta(0)=0$ :

$$
\begin{aligned}
\xi & =-\eta^{2} y_{0}(0) \\
c \beta+k \gamma & =-\eta \theta_{0}(0)
\end{aligned}
$$

\footnotetext{
2 this is a simplification that is not always performed in the SCE method.
} 


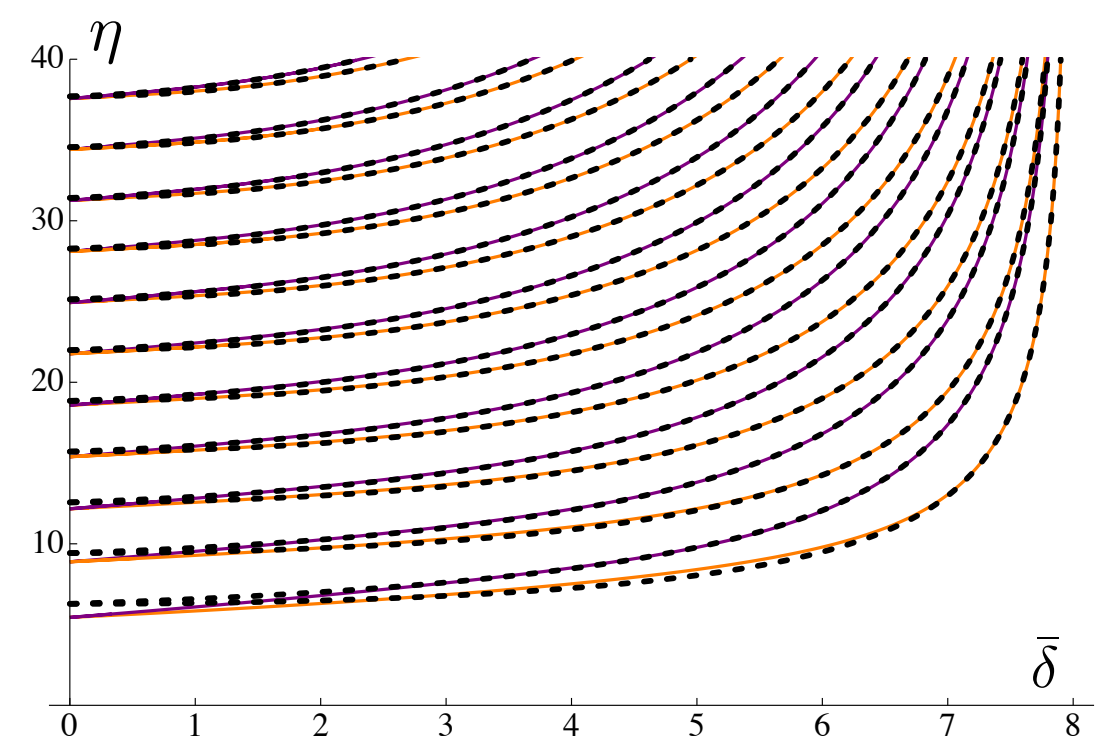

Figure 9: Curves $M$ and $N$ separating the regions of the phase diagram of Fig. 7. Continuous curves, redrawn from Fig. 7, are obtained numerically while dashed curves are plotted using the asymptotic results (33) and (34).

The other two coefficients are found using the symmetry conditions at $s=1 / 2$. For symmetric (respectively antisymmetric) configurations we have $\theta(1 / 2)=\theta^{\prime \prime}(1 / 2)=0$ (resp. $\left.y(1 / 2)=\theta^{\prime}(1 / 2)=0\right)$. As the homoclinic solution $(23)$ automatically satisfies these symmetry conditions we simply require $\theta_{1}=\theta_{1}^{\prime \prime}=0$ (symmetric solution), or $y_{1}=\theta_{1}^{\prime}=0$, at $\tilde{s}=\eta / 2$. We use the approximations $\cosh (c \eta / 2) \simeq \sinh (c \eta / 2) \simeq(1 / 2) e^{c \eta / 2}$ as $\eta \gg 1$ and eventually arrive at:

$$
\begin{aligned}
& \alpha=8 c \eta\left[\frac{1+c^{2}}{k^{2}} \sin (k \eta[\phi-1 / 2])-\frac{c}{k} \cos (k \eta[\phi-1 / 2])\right] \mathrm{e}^{-c \eta / 2} \\
& \beta=8 c \eta\left[\frac{c}{k} \sin (k \eta[\phi-1 / 2])-\cos (k \eta[\phi-1 / 2])\right] \mathrm{e}^{-c \eta / 2} \\
& \gamma=-\alpha \\
& \xi=-\beta
\end{aligned}
$$

As soon as $\eta \gtrsim 15$, the asymptotic expansion (24) with (23), 27]), and (30) uniformly matches the numerical solution, as shown in Fig. 8

\subsection{Loci of points $\mathrm{M}$ and $\mathrm{N}$}

We look for an approximation for the loci of points $M$ and $N$ introduced in Section 4.3 These points are defined as $\theta^{\prime}(0)=0$ and $\theta^{\prime \prime}(0)=0$ respectively. We use the asymptotic expansion to calculate

$$
\begin{aligned}
\theta^{\prime}(0) & \simeq \frac{32 c^{2} \eta}{k} \mathrm{e}^{-c \eta / 2} \sin (k \eta[\phi-1 / 2]) \\
\theta^{\prime \prime}(0) & \simeq \frac{32 c^{2} \eta^{2}}{k} \mathrm{e}^{-c \eta / 2}[c \sin (k \eta[\phi-1 / 2])-k \cos (k \eta[\phi-1 / 2])]
\end{aligned}
$$

and therefore find that

$$
\begin{aligned}
& \mathrm{M}: \eta \simeq \frac{2 i \pi+\chi \pi}{k} \quad, i=1,2,3, \ldots \\
& \mathrm{N}: \eta \simeq \frac{2(i+1) \pi-\chi \pi-2 \arctan (k / c)}{k} \quad, i=1,2,3, \ldots
\end{aligned}
$$


with $\chi=0$ (respectively $\chi=1$ ) for symmetric (resp. anti-symmetric) configurations. As $k=\left[1-(\bar{\delta} / 8)^{2}\right]^{\frac{1}{2}}$ and $c=\bar{\delta} / 8$ [25], (33) and (34) define curves in the plane $(\bar{\delta}, \eta)$. In Fig. 9 we plot these curves (dashed lines) and compare them to those obtained numerically (continuous line) and already displayed in Fig. 7. We see that a very good agreement is obtained for as soon as $\eta>10$. The asymptotic expansion allows us to extend the numerical predictions far above the limits of the numerical algorithms. As a matter of fact, it is difficult to perform numerical continuation for $\eta>30$, while the asymptotic expansion is more and more accurate as $\eta$ grows.

Note that the case of non-symmetric solutions remains to be treated. In this case a supplementary shifting parameter is needed for the homoclinic solution $\theta_{0}$ [27] and an expansion near $s=1$ has to be added to $\theta_{1}$.

\section{Conclusion}

In this paper we have studied the equilibrium shapes of a finite-length elastic beam buckling on a liquid foundation and we have computed the bifurcation paths of equilibrium solutions in the post-buckling regime. In this problem the buckling mode can be either symmetric or anti-symmetric, depending on the length of the beam. We have found that the switching from symmetric to anti-symmetric (and vice versa) buckling modes triggers the appearance of secondary bifurcations in the post-buckling domain, where special branches connect symmetric and anti-symmetric equilibrium paths. The number of connection branches increases with the length of beam. All the solutions along the connection branches are stable and non-symmetric. Therefore as the confinement rate of the beam is increased we observe an alternation of symmetric and anti-symmetric shapes, connected by non-symmetric transitions. This phenomenon is called mode branching and is typical of beam buckling on stiffening foundations. Moreover, as the function $\theta(s)$ loses one zero at every passage through a connection branches, the sinusoidal pattern observed at buckling localizes into a well-defined fold, leaving the beam flat elsewhere. Secondary bifurcations are an essential ingredient in this route to localization. Note that localization is a typical feature of beam buckling on softening foundations. It thus appears that the liquid foundation combines post-buckling responses of both stiffening and softening foundations. The number of non-symmetric, stable regions clearly increases with $\eta$ (Fig. 7). Eventually, this leads to the continuous symmetry of the solution for the homoclinic $(\eta \rightarrow \infty)$ case. While no clear indication exists on the stability of the non-symmetric profiles of the homoclinic solution, we have here demonstrated that non-symmetric solutions are stable in the finite $\eta$ case.

\section{Acknowledgments}

MR thanks Enrique Cerda for useful discussions about his previous theoretical and experimental work on folding of floating sheets. The present work was supported by ANR grant ANR-09-JCJC-0022-01. Financial support from 'La Ville de Paris - Programme Émergence' is also gratefully acknowledged.

\section{A Match Asymptotic Expansion for the case $\eta \gg 1$}

We here show the resolution of the problem of Section 5 using the method of matched asymptotic expansions (MAE). For simplicity we only treat the case of a symmetrical solution and study the solution in $s \in(0,1 / 2)$. We identify two regions: $(i)$ an inner region $s \in\left(0, \epsilon s_{1}\right)$ where $\theta(s)$ is small, and $(i i)$ an outer region $s \in\left(\epsilon s_{2}, 1 / 2\right)$ where $\theta(s)=O(1)$, with $s_{1}$ and $s_{2}$ being $O(1)$. The small variable $\epsilon$ is defined as $\epsilon=1 / \eta$. In each region $\theta(s)$ has to fulfill approximatively the differential equation (7) but with different boundary conditions, that is the function $\theta(s)$ will be approximated by different expansions which will have to match in the intermediate region $s \sim \epsilon s_{1} \sim \epsilon s_{2}$. As in Section 5 we use the approximation $\mathcal{H} \simeq-p$.

\section{The outer region}

Here we set $\theta(s) \simeq \theta_{0}(s)$, given by 23 a with $\phi=0$. Symmetry conditions at $s=1 / 2, \theta(1 / 2)=\theta^{\prime \prime}(1 / 2)=0$, are automatically fulfilled. Anticipating the matching, we develop $\theta_{0}(s)$ for $s \rightarrow 0$, using the variable $\tilde{s}=\eta s$. As $\theta_{0}(s)$ 

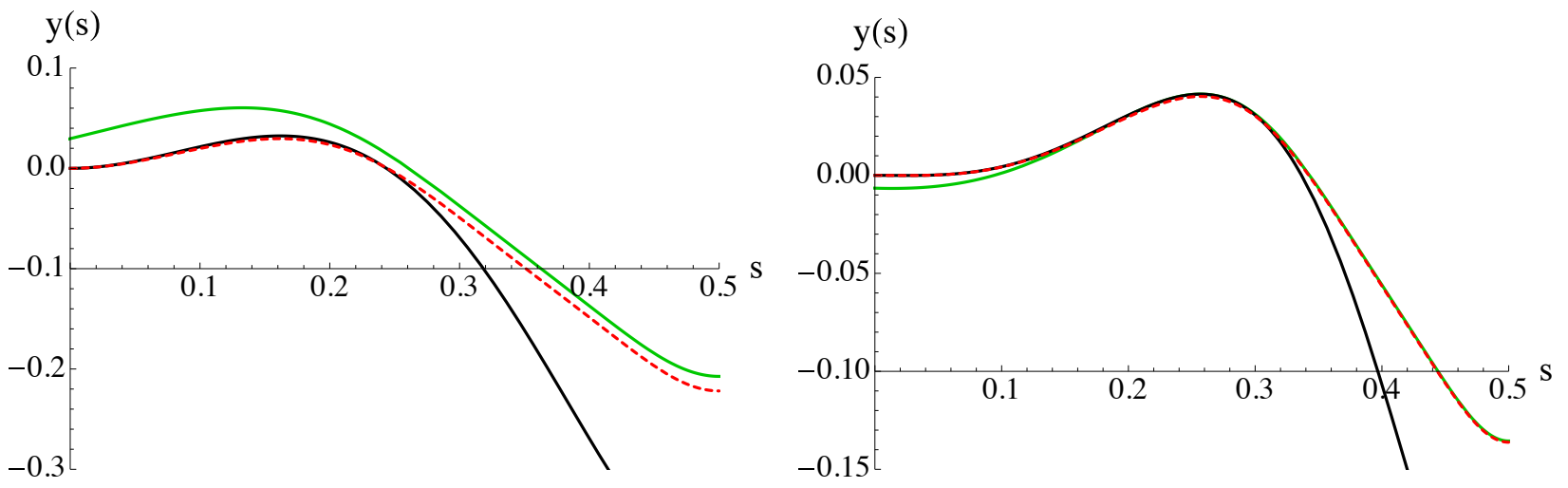

Figure 10: The deflection $y(s)$ for the same symmetrical solutions as in Fig. 8: comparison between $(i)$ the numerical resolution (red dashed curve), (ii) the outer solution (23) (green curve), and (iii) the inner soution (37) (black curve), for $\bar{\delta}=4$ and $\eta=10$ (left), $\eta=15$ (right). The outer solution approximates the numerical solution in the region $s \sim 0.5$ but fails to do so in the region $s \sim 0$. Respectively the inner solution approximates the numerical solution in the region $s \sim 0$ but fails to do so in the region $s \sim 0.5$.

becomes small, we have $\theta_{0}(s) \simeq 4(c / k) \operatorname{sech}(c \tilde{s}-c \eta / 2) \sin (k \tilde{s}-k \eta / 2)$. Using $\operatorname{sech}(-X) \simeq 2 e^{-X}$ for $X \gg 1$ and developing the sinus, we arrive at

$$
\theta_{\text {outer }} \simeq \frac{\mathrm{e}^{c \tilde{s}}}{2}(A \sin k \tilde{s}+B \cos k \tilde{s})
$$

with $A=16(c / k) \cos (k \eta / 2) \mathrm{e}^{-c \eta / 2}$ and $B=-16(c / k) \sin (k \eta / 2) \mathrm{e}^{-c \eta / 2}$.

\section{The inner region}

Here $\theta(s)$ is small and found to be $O(\epsilon)$. We set $\theta(s) \simeq \epsilon \theta_{1}(s)$ and we linearize Eq. (7) to $\theta_{1}^{\prime \prime \prime \prime}+p \theta_{1}^{\prime \prime}+\eta^{4} \theta_{1}=0$. Using the magnification $\tilde{s}=s / \epsilon$, as defined previously, we arrive at an equation where $\epsilon$ no longer appears:

$$
\dddot{\theta_{1}}+\bar{p} \ddot{\theta}_{1}+\theta_{1}=0
$$

where, as before, $\bar{p}=\epsilon^{2} p$ and $\left(\dot{)}=\mathrm{d}() / \mathrm{d} \tilde{s}\right.$. We then look for the solution $\theta_{1}(\tilde{s})=\dot{y}_{1}(\tilde{s})$ with

$$
y_{1}(\tilde{s})=\alpha_{1} \sinh (c \tilde{s}) \sin (k \tilde{s})+\beta_{1} \sinh (c \tilde{s}) \cos (k \tilde{s})+\gamma_{1} \cosh (c \tilde{s}) \sin (k \tilde{s})+\xi_{1} \cosh (c \tilde{s}) \cos (k \tilde{s})
$$

and $y(s) \simeq \epsilon^{2} y_{1}(s)$. Note that the coefficients $\left(\alpha_{1}, \beta_{1}, \gamma_{1}, \xi_{1}\right)$ will be different from the coefficients $(\alpha, \beta, \gamma, \xi)$ found in Section 5 . The boundary conditions $\theta_{1}(0)=0$ and $y_{1}(0)=0$ impose $\gamma_{1}=-c \beta_{1} / k$ and $\xi_{1}=0$, yielding:

$$
\theta_{1}(\tilde{s})=c \alpha_{1} \cosh (c \tilde{s}) \sin (k \tilde{s})+k \alpha_{1} \sinh (c \tilde{s}) \cos (k \tilde{s})-\left(\beta_{1} / k\right) \sinh (c \tilde{s}) \sin (k \tilde{s})
$$

The two remaining coefficients $\alpha_{1}$ and $\beta_{1}$ are to be found with the matching procedure.

\section{Matching the inner and outer solutions}

We develop 38 for large $\tilde{s}$ using $\cosh (X) \simeq \sinh (X) \simeq(1 / 2) e^{X}$ when $X \gg 1$ and find:

$$
\theta_{\text {inner }} \simeq \frac{\mathrm{e}^{c \tilde{s}}}{2}\left(\left[c \alpha_{1}-\frac{\beta_{1}}{k}\right] \sin k \tilde{s}+k \alpha_{1} \cos k \tilde{s}\right)
$$

Identifying (35) and $(39)$ we find

$$
\begin{aligned}
& \alpha_{1}=-16 \eta\left(c / k^{2}\right) \mathrm{e}^{-c \eta / 2} \sin (k \eta / 2) \\
& \beta_{1}=-16 \eta(c / k) \mathrm{e}^{-c \eta / 2}[k \cos (k \eta / 2)+c \sin (k \eta / 2)]
\end{aligned}
$$


We first remark that, as in any boundary layer approach, each solution is only valid in its own region, see Fig. 10 . and that a uniformly valid approximation has yet to be found. Nevertheless, as solution $(38)$ is valid near $s=0$, we have approximations to $\theta^{\prime}(0)$ and $\theta^{\prime \prime}(0)$ that can be compared to those found in Section 5.2 Using $\theta(s) \simeq \epsilon \theta_{1}(s / \epsilon)$ and $(38)$, we calculate $\theta^{\prime}(0) \simeq 2 c k \alpha_{1}$ and $\theta^{\prime \prime}(0) \simeq 2 c \eta \beta_{1}$, which indeed corresponds to (31) et (32).

\section{References}

[1] Stephen P. Timoshenko. Strength of Materials. D. van Nostrand, New York, 1940.

[2] E. Cerda and L. Mahadevan. Geometry and physics of wrinkling. Phys. Rev. Lett., 90:074302, 2003.

[3] Kirill Efimenko, Mindaugas Rackaitis, Evangelos Manias, Ashkan Vaziri, L. Mahadevan, and Jan Genzer. Nested self-similar wrinkling patterns in skins. Nat Mater, 4(4):293-297, 042005.

[4] Luka Pocivavsek, Robert Dellsy, Andrew Kern, Sebastián Johnson, Binhua Lin, Ka Yee C. Lee, and Enrique Cerda. Stress and fold localization in thin elastic membranes. Science, 320(5878):912-916, 2008.

[5] Shengqiang Cai, Dayong Chen, Zhigang Suo, and Ryan C. Hayward. Creasing instability of elastomer films. Soft Matter, 8:1301-1304, 2012.

[6] Dominic Vella, José Bico, Arezki Boudaoud, Benoit Roman, and Pedro M. Reis. The macroscopic delamination of thin films from elastic substrates. Proceedings of the National Academy of Sciences, 106(27):10901-10906, 2009.

[7] Till J. W. Wagner and Dominic Vella. Floating carpets and the delamination of elastic sheets. Physical Review Letters, 107(4):044301, 072011.

[8] S. T. Milner, J. F. Joanny, and P. Pincus. Buckling of langmuir monolayers. EPL (Europhysics Letters), 9(5):495, 1989.

[9] Svetlana Baoukina, Luca Monticelli, H. Jelger Risselada, Siewert J. Marrink, and D. Peter Tieleman. The molecular mechanism of lipid monolayer collapse. Proceedings of the National Academy of Sciences, 105(31):1080310808, 2008.

[10] Miguel Trejo, Carine Douarche, Virginie Bailleux, Christophe Poulard, Sandrine Mariot, Christophe Regeard, and Eric Raspaud. Elasticity and wrinkled morphology of bacillus subtilis pellicles. Proceedings of the National Academy of Sciences, 110(6):2011-2016, 2013.

[11] E. P. Chan, E. J. Smith, R. C. Hayward, and A. J. Crosby. Surface wrinkles for smart adhesion. Advanced Materials, 20(4):711-716, 2008.

[12] P. M. Reis, F. Corson, A. Boudaoud, and B. Roman. Localization through surface folding in solid foams under compression. Phys. Rev. Lett., 103:045501, Jul 2009.

[13] Ned Bowden, Scott Brittain, Anthony G. Evans, John W. Hutchinson, and George M. Whitesides. Spontaneous formation of ordered structures in thin films of metals supported on an elastomeric polymer. Nature, 393:146-149, 1998.

[14] D. Vella, P. Aussillous, and L. Mahadevan. Elasticity of an interfacial particle raft. Europhys. Lett., 68(2):212-218, 2004.

[15] E. P. Chan and A. J. Crosby. Fabricating microlens arrays by surface wrinkling. Advanced Materials, 18(24):3238$3242,2006$.

[16] John A. Rogers, Takao Someya, and Yonggang Huang. Materials and mechanics for stretchable electronics. Science, 327(5973):1603-1607, 2010. 
[17] A. R. Champneys, G. W. Hunt, and J. M. T. Thompson. Localization and solitary waves in solid mechanics. Phil. Trans. R. Soc. Lond. A, 355:2077-2081, 1997.

[18] Michel Potier-Ferry. Amplitude modulation, phase modulation and localization of buckling patterns. In J. M. T. Thompson and G. W. Hunt, editors, Collapse: The Buckling of Structures in Theory and Practice, pages 149-159. Cambridge University Press, 1983.

[19] G. W. Hunt, M. K. Wadee, and N. Shiacolas. Localized elasticae for the strut on the linear foundation. Journal of Applied Mechanics, 60:1033-1038, 1993.

[20] G. W. Hunt, H. M. Bolt, and J. M. T. Thompson. Structural localization phenomena and the dynamical phase-space analogy. Proceedings of the Royal Society of London. Series A, Mathematical and Physical Sciences, 425(1869):245-267, 1989

[21] Fabian Brau, Hugues Vandeparre, Abbas Sabbah, Christophe Poulard, Arezki Boudaoud, and Pascal Damman. Multiple-length-scale elastic instability mimics parametric resonance of nonlinear oscillators. Nat Phys, 7(1):5660, 012011.

[22] Fabian Brau, Pascal Damman, Haim Diamant, and Thomas A. Witten. Wrinkle to fold transition: influence of the substrate response. Soft Matter, 9:8177-8186, 2013.

[23] B. Audoly. Localized buckling of a floating elastica. Physical Review E, 84(1):011605, 072011.

[24] Alan R. Champneys. Homoclinic orbits in reversible systems and their applications in mechanics, fluids and optiks. Physica D: Nonlinear Phenomena, 112:158-186, 1998.

[25] Haim Diamant and Thomas A. Witten. Compression induced folding of a sheet: An integrable system. Physical Review Letters, 107(16):164302, 102011.

[26] Marco Rivetti. Non-symmetric localized fold of a floating sheet. Comptes Rendus Mécanique, 341(3):333 - 338, 2013.

[27] Haim Diamant and Thomas A. Witten. Shape and symmetry of a fluid-supported elastic sheet. Phys. Rev. E, 88:012401, 2013.

[28] S. Neukirch, G.H.M. van der Heijden, and J.M.T. Thompson. Writhing instabilities of twisted rods: from infinite to finite length. Journal of the Mechanics and Physics of Solids, 50(6):1175 - 1191, 2002.

[29] Marco Rivetti and Arnaud Antkowiak. Elasto-capillary meniscus: pulling out a soft strip sticking to a liquid surface. Soft Matter, 9:6226-6234, 2013.

[30] A. E. H. Love. A Treatise on the Mathematical Theory of Elasticity. Dover Publications, New York, 4th edition, 1944.

[31] G. Domokos. Global description of elastic bars. Z. Angew. Math. Mech, 74:T289-T291, 1994.

[32] M. Stein. The phenomenon of change in buckle pattern in elastic structures. Technical Report R39, NASA, 1959.

[33] W.J. Supple. Coupled branching configurations in the elastic buckling of symmetric structural systems. International Journal of Mechanical Sciences, 9(2):97 - 112, 1967.

[34] P.R. Everall and G.W. Hunt. Mode jumping in the buckling of struts and plates: a comparative study. International Journal of Non-Linear Mechanics, 35(6):1067-1079, 2000.

[35] Jean Cousteix and Jacques Mauss. Asymptotic analysis and boundary layers. Springer, 2007. 